\title{
Assessment of Molecular Signalling Mechanisms for Eosinophilia in Rottweilers
}

\author{
Nathalee Prakash ${ }^{1,2}$, Michael J. Day ${ }^{3}$, Phil Stumbles ${ }^{1}$, Iain R. Peters ${ }^{3}$, Mellora Sharman ${ }^{1,2}$, \\ Amanda Paul ${ }^{1}$, Caroline Mansfield ${ }^{1,2}$ \\ ${ }^{1}$ School of Veterinary and Biomedical Sciences, Murdoch University, Perth, Australia \\ ${ }^{2}$ Faculty of Veterinary Science, The University of Melbourn, Parkville, Australia \\ ${ }^{3}$ School of Veterinary Sciences, University of Bristol, Bristol, UK \\ Email: cmans@unimelb.edu.au
}

Received August 23, 2012; revised September 29, 2012; accepted October 9, 2012

\begin{abstract}
Rottweilers are predisposed to eosinophilic diseases, including hypereosinophilic syndrome. The immunopathogenesis of idiopathic eosinophilia is poorly characterised in dogs and man. Studies in people have suggested cytokines, particularly interleukin (IL)-5, play a role in instigating and perpetuating eosinophilia. This study sought to establish whether differences in gene expression, and concentration of selected, cytokines and chemokines were associated with eosinophilia in Rottweilers. Quantitative real-time reverse transcriptase polymerase chain reaction (qRT-PCR) assays were used to quantify messenger ribonucleic acid (mRNA) encoding cytokines IL-4, -5, -10, -12p19, -12p35, -12p40, -18, interferon gamma (IFN- $\gamma$ ) and chemokines eotaxin-2 and -3 from peripheral blood mononuclear cell (PMBC) samples obtained from healthy dogs (breeds other than Rottweiler) with normal eosinophil blood counts $(n=5)$ and Rottweilers with normal $(n=6)$, mildly increased $(n=7)$ and high $(n=3)$ eosinophil blood counts. Quantification of plasma IFN- $\gamma$ and IL-5 was performed using commercially available canine-specific enzyme-linked immunosorbent assays ELISAs. Cytokine mRNA was measurable in all samples, although eotaxin-2 and -3 were not detected. No significant differences in gene expression of any cytokine were found between groups (based on eosinophil count or breed). No significant difference in plasma IL-5 or IFN- $\gamma$ concentration was present between groups. In conclusion, there were no significant differences in cytokine mRNA profiles or plasma IL-5 and IFN- $\gamma$ levels between Rottweilers with increased eosinophil counts and Rottweiler and non-Rottweiler dogs with normal eosinophil counts.
\end{abstract}

Keywords: Cytokines; Dogs; IL-5; IFN- $\gamma$; Hypereosinophilic Syndrome

\section{Introduction}

Eosinophils play an important role in innate immunity, inflammation and homeostatic host responses in dogs [1]. Eosinophil granules contain highly cationic proteins with cytotoxic and pro-thrombotic properties, and these cells are known to incite endothelial damage [1]. In particular, eosinophils can produce substances such as platelet activating factor that can alter bronchial and vascular smooth muscle tone, modify vascular permeability and stimulate chemotaxis of other inflammatory cells $[1,2]$. Eosinophils respond to different stimuli, particularly parasitic infection, where their cellular products can be protective to the host [3]. However, exuberant release of cellular products by eosinophils can contribute to tissue damage, particularly in the respiratory and gastrointestinal tracts $[4,5]$.

Idiopathic eosinophilic diseases (or eosinophilic infiltration in the absence of an identifiable parasitic or allergic trigger) have been described in multiple breeds such as German shepherd dogs, Cavalier King Charles spaniels, Siberian Huskies, Alaskan Malamutes and Rottweilers [3-11]. In many reports, Rottweilers appear to be overrepresented with idiopathic eosinophilic disease [4]. It has been postulated that Rottweilers may have an exaggerated response to allergic stimuli or parasite exposure $[4,12]$.

Studies in people with eosinophilic disorders have highlighted the role of cytokines, particularly interleukin (IL)-5, in mediating eosinophil release [13,14]. IL-5 forms part of the type-2 (Th2) cytokine profile, and is essential for eosinophil maturation, differentiation and egress from the bone marrow [1,2]. In people with eosinophilic disorders, exaggerated release of IL-5 by reactive eosinophils has been demonstrated in vivo [5]. The chemokines eotaxin-2 (CCL24) and eotaxin-3 (CCL26) are also able to induce chemotaxis and release of reactive oxygen species in eosinophils via interaction with chemokine receptor CCR3 [15-18]. It is unknown what cellular 
signalling drives the production of hypereosinophilic syndrome (HES) and other eosinophilic disease in dogs, or why Rottweilers are predisposed.

The aim of this study was to establish if there are definable differences in expression of mRNA encoding cytokines and eotaxins, or in plasma concentrations of IL-5 and IFN- $\gamma$ in Rottweilers with variable eosinophil counts and healthy dogs of other breeds.

\section{Materials and Methods}

\subsection{Animals}

The study was conducted with full Murdoch University Animal Ethics Committee approval, based on NHMRC guidelines, with signed owner consent obtained. Dogs were identified upon presentation to Murdoch University Veterinary Hospital, as well as from Rottweilers identified from a haematological survey of breeding kennels performed previously (Mansfield, unpublished observations 2004). Each dog had a full physical examination, with particular focus on the detection of ectoparasites and evidence of allergic skin disease. Dogs with eosinophilia were further assessed for common aetiologies via faecal floatation, repeat haematology, heartworm antigen testing, skin scraping and an anthelminthic trial. Further tests, such as radiography, ultrasonography, adrenocorticotrophic (ACTH)-stimulation tests, fine-needle aspiration of affected organs, bone marrow biopsy or endoscopy, were performed on an individual basis, determined by the animals' degree of eosinophilia, physical examination findings and localising clinical signs.

\subsection{Collection and Processing of Material}

Whole blood was obtained via cephalic or jugular venipuncture into a $2.5 \mathrm{~mL}$ EDTA container and three 4.5 $\mathrm{mL}$ lithium heparin containers. A complete blood count (CBC) was performed using an automated haematology analyser (Advia, Siemens, Germany) and confirmed by a manual differential. Heparinised blood samples were processed by a 1:1 dilution with physiological saline followed by density gradient centrifugation (Lymphoprep, Axis-Shield, Norway), to isolate peripheral blood mononuclear cells (PBMC). The pelleted PBMC were washed, resuspended in freezing medium (foetal calf serum with $10 \%$ dimethyl sulfoxide) and stored in liquid nitrogen until shipped on dry ice for RNA isolation.

\subsection{RNA Isolation}

Total RNA was isolated from PBMC using a Macherey-Nagel NucleoSpin RNA II Isolation Kit (ABgene, Epsom, UK). Further deoxyribonuclease (DNase) digestion of the RNA solution was performed using RQ1 Ri-
bonuclease-Free DNase (Promega Corporation, Southampton, UK), as per the manufacturer's instructions. Quantity and quality of resulting RNA was assessed by automated gel electrophoresis (Experion, Bio-Rad Laboratories, Hemel Hempstead, UK) prior to storage at $-70^{\circ} \mathrm{C}$.

\subsection{Primer and Hydrolysis Probe Design}

Primers and probes for chemokines and cytokines were designed using Primer 3 [19] and MFold, from the canine specific GenBank sequences for IL-12p35 (accession number: U49085) and IL-12p19 (accession number: XM_538231) as described by Peters et al. (2004) (Table 1) [19]. IL-4, -5, -10, -12p19, -12p35, -12p40, -18, interferon (IFN)- $\gamma$, eotaxin-2 and eotaxin-3 specific assays have previously been validated in dogs [20-24]. Assays for potential housekeeper genes glyceraldehyde-3-phosphate dehydrogenase (G3PDH), hydroxylmethylbilane synthase (HMBS), hypoxanthine phosphoribosyl-transferase 1 (HPRT1), ribosomal protein L13a (RPL13A), ribosomal protein S18 (RPS18), succinate dehydrogenase complex, subunit A (SDHA), TATA box binding protein (TBP), tyrosine 3-monooxygenase/tryptophan 5-monooxygenase activation protein and zeta polypeptide (YWAZ) were performed as described by Peters et al. (2007) [24] (Supplementary Table A1).

\subsection{Quantitative RT-PCR}

Synthesis of complimentary DNA (cDNA) was performed using 500ng of random hexamers with the ImProm-II Reverse Transcription System (Promega Corporation, Madison, WI), according to the manufacturer's instructions. Quantitative RT-PCR was performed using HotStarTaq Master Mix (Qiagen Ltd., Crawley, UK), with sample incubations performed in an iCycler IQ (Bio-Rad Laboratories Ltd., Hercules, CA) during which fluorescence data were collected. The absence of genomic contamination of the RNA samples and nuclease-free water was confirmed prior to the reverse transcriptase reactions and none of the samples showed evidence of amplifiable genomic DNA with the G3PDH qPCR assay.

In order to determine the most appropriate housekeeper genes for the study, all 8 potential genes were quantified in all cDNA samples. A mean cycle threshold (Ct) value was calculated for each PBMC sample using the two measured $\mathrm{Ct}$ values. The mean $\mathrm{Ct}$ value was converted to a relative copy number value using the $\mathrm{E}^{\Delta \mathrm{Ct}}$ method (E: reaction efficiency) with $\Delta \mathrm{Ct}$ values calculated relative to the sample with the largest $\mathrm{Ct}$ (fewest gene copies). The geNorm VBA applet for Microsoft Excel was used to determine the level of stability of the test genes [25]. 
Relative expression data for each of the gene cytokine/ chemokine targets was calculated using the gBase applet for Microsoft Excel (http://medgen.ugent.be/qbase/), normalised against the 3 most stable housekeeper genes. The sample with the fewest gene copies (latest $\mathrm{Ct}$ value) was given a relative copy number of 1 and all other samples were given values relative to this sample. These relative copy number results were used for the statistical testing and presentation in graphical form.

\subsection{Quantification of IFN- $\gamma$ and IL-5 Using ELISA}

IFN- $\gamma$ and IL-5 concentrations were measured in heparinised plasma samples with canine-specific ELISA kits for IFN- $\gamma$ (R\&D Systems, Minneapolis, USA) and IL-5 (USCN Life Science Inc., USA), according to manufacturer's instructions.

\subsection{Statistical Analysis}

Dogs were categorised as clinically healthy Rottweilers with normal eosinophil counts ("RottL"; $\leq 1.0 \times 10^{9} / \mathrm{L}$ ); clinically healthy adult Rottweilers with mild increase in eosinophil counts ("RottM"; $1.0-2.0 \times 10^{9} / \mathrm{L}$ ); Rottweilers that had a moderate to severe increase in eosinophil counts $\left(\geq 2.0 \times 10^{9} / \mathrm{L}\right)$ ("RottH"); and dogs of other breeds with normal eosinophil counts ("N").

For each cytokine, a Kruskal-Wallis analysis of variance and Mann Whitney $U$-test were performed to examine differences in relative copy numbers obtained in the plasma samples between the groups RottH, RottM, RottL and N, between RottL and N, and between RottH + RottM + RottL and N, as well as between RottH + RottM and Rott L and N. As the results were not normally distributed (using a Kolmogorov-Smirnov test), non-parametric testing was used to examine differences between the groups for each cytokine. The results of each group for IL-5 and IFN- $\gamma$ concentrations were compared using unpaired $t$-tests with unequal variances. For all analyses, differences were considered significant when $P$ $<0.05$.

The correlation coefficient was calculated to investigate the relation between IL-5 concentration and mRNA.

\section{Results}

\subsection{Animals}

There were a total of 21 dogs recruited for the study. There were 6 clinically healthy Rottweilers with normal eosinophil counts ("RottL"), 7 clinically healthy adult Rottweilers with increased eosinophil counts ("RottM"), 3 Rottweilers with hypereosinophilic disease ("RottH"); and 5 healthy dogs of other breeds with normal eosino- phil counts ("N"). The other breeds included 1 English springer spaniel, 2 Whippets, 1 Greyhound and a Labrador crossbreed dog. The mean age of Rottweilers in groups RottM and RottH was 2.5 years (range 1 to 4 years), compared to a mean age of 3.27 years (range 1 to 6 years) of dogs in RottL and $\mathrm{N}$ respectively. There were four males (three entire) and one female (neutered) in $\mathrm{N}$, four females (all entire) and two males (both neutered) in RottL, five females (one neutered) and two males (both entire) in RottM, and one neutered male and two females (one neutered, one entire) in RottH. The majority of Rottweilers were breeding animals kept in kennel situations.

\subsection{Quantitative RT-PCR}

Transcripts of cytokines IL-4, $-5,-10,-12 \mathrm{p} 19,-12 \mathrm{p} 35$, $-12 \mathrm{p} 40,-18$, and IFN- $\gamma$ could be quantified in all of the samples, but mRNA encoding eotaxins- 2 and -3 were not detected in any sample. All samples were positive for the housekeeper genes tested. RPL13A, SDHA and YWAZ were selected as the most stably expressed genes for use in normalisation of the expression data (Table 1).

Plots of relative copy numbers for IL-5 and IFN- $\gamma$ are shown in Figures 1 and 2, respectively. There were no significant differences in mRNA encoding between the groups for any cytokine. Despite a tendency for IFN- $\gamma$ mRNA expression to be lower in RottH/RottM versus RottL/N, this did not reach statistical significance $(P=$ $0.062)$.

\subsection{Quantification of IFN- $\gamma$ by ELISA}

The trend for decreased IFN- $\gamma$ mRNA expression in

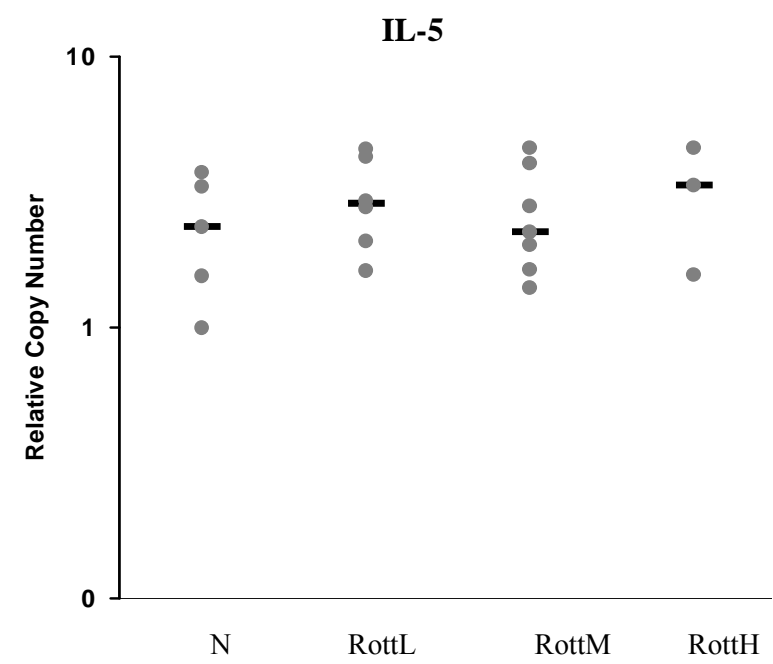

Figure 1. Plots of the relative copy number from QRT-PCR assays for IL-5. The horizontal line corresponds to the median value of that group. There were no significant differences in the transcription of mRNA encoding IL-5 between the groups. 


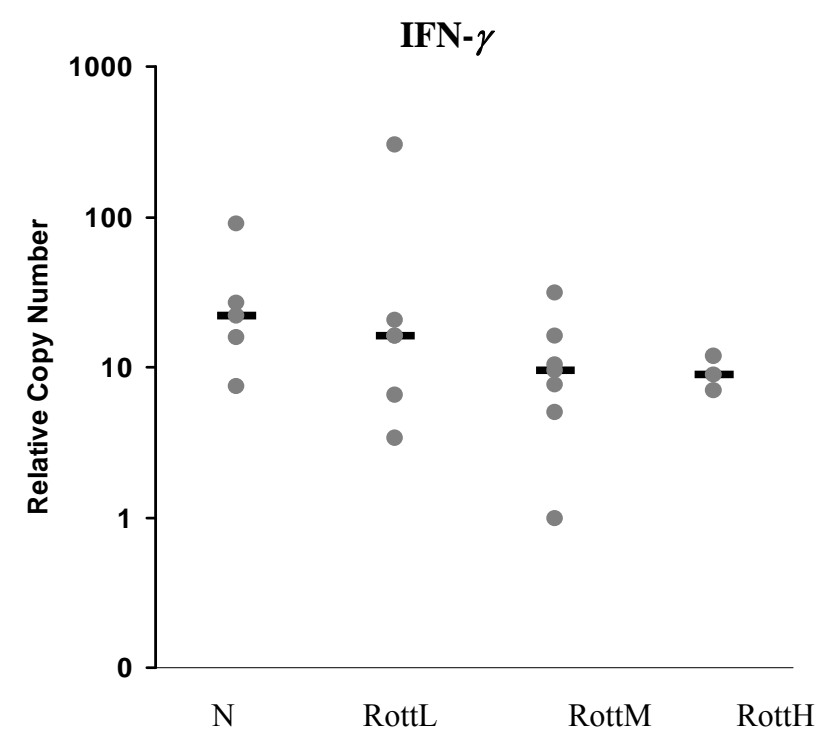

Figure 2. Plots of the relative copy number from QRT-PCR assays for IFN- $\gamma$. The horizontal line corresponds to the median value of that group. There were no significant differences in the transcription of mRNA encoding IFN- $\gamma$ between the groups, although there was a tendency for IFN- $\gamma$ mRNA expression to be lower in the diseased group versus the control dogs.
Rottweilers with increased eosinophil count did not correlate with changes in the plasma concentration of IFN- $\gamma$. The majority of dogs had IFN- $\gamma$ concentrations below the minimum detectable level of the assay (Figure 3). Only $3 / 7$ dogs from group RottM had measurable IFN- $\gamma$ concentrations $(16.45,48.71$, and $51.47 \mathrm{pg} / \mathrm{mL})$. There were no significant differences between normal and diseased animals in the concentration of plasma IFN $-\gamma$.

\subsection{Quantification of IL-5 by ELISA}

Plasma IL-5 was detectable in 11 dogs. The median concentrations of IL-5 concentrations detected in RottH + RottM versus RottL+N were $14.325 \mathrm{pg} / \mathrm{mL}$ and 0.0 $\mathrm{pg} / \mathrm{mL}$ respectively. The median concentrations of RottL + RottM + RottH versus non-Rottweiler dogs were 11.36 $\mathrm{pg} / \mathrm{mL}$ and $14.09 \mathrm{pg} / \mathrm{mL}$ respectively. There were no significant differences between RottH + RottM versus RottL + N, or between breeds (Figure 4).

\subsection{Correlation of Quantification of IL-5 and IFN- $\gamma$ mRNA and Protein Quantified by ELISA}

In this study, plasma IL-5 and IFN- $\gamma$ measured by ELISA

Table 1. Results of quantification of transcripts for IL-4, IL-5, IL-10, IL-12p19, IL-12p35, IL-12p40, IL-18, and IFN- $\gamma$ : All results were expressed relative to the sample with the fewest number of gene copies for each target (not always the same sample for different genes).

\begin{tabular}{|c|c|c|c|c|c|c|c|c|c|}
\hline Sample type & $\begin{array}{c}\text { Absolute } \\
\text { eosinophils }\end{array}$ & IL-4 & IL-5 & IL-10 & IL-12p19 & IL-12p35 & IL-12p40 & IL-18 & IFN- $\gamma$ \\
\hline $\mathrm{N}$ & 0 & 1.0 & 3.8 & 2.6 & 1.8 & 2.1 & 1.8 & 1.0 & 15.7 \\
\hline $\mathrm{N}$ & 0.2 & 9.4 & 1.0 & 3.0 & 2.1 & 1.0 & 3.4 & 1.9 & 22.0 \\
\hline $\mathrm{N}$ & 0.3 & 9.4 & 2.4 & 4.3 & 2.0 & 3.2 & 5.5 & 3.8 & 7.5 \\
\hline $\mathrm{N}$ & 0.1 & 24.7 & 1.6 & 1.0 & 1.0 & 1.4 & 1.0 & 3.9 & 90.5 \\
\hline $\mathrm{N}$ & 0.1 & 6.2 & 3.3 & 3.4 & 3.2 & 1.5 & 5.3 & 3.4 & 26.9 \\
\hline RottL & 0.3 & 20.9 & 4.3 & 1.2 & 3.3 & 1.2 & 5.1 & 1.4 & 6.6 \\
\hline RottL & 0.5 & 8.4 & 2.8 & 2.3 & 2.2 & 3.7 & 4.7 & 5.3 & 301.8 \\
\hline RottL & 0.6 & 4.9 & 1.6 & 2.3 & 1.5 & 2.0 & 3.8 & 2.4 & 16.1 \\
\hline RottL & 1 & 12.0 & 4.6 & 1.9 & 2.2 & 1.5 & 2.1 & 5.4 & 16.1 \\
\hline RottL & 0.6 & 5.9 & 3.0 & 2.3 & 2.6 & 1.5 & 2.4 & 3.1 & 3.4 \\
\hline RottL & 0.7 & 10.2 & 2.1 & 2.0 & 1.5 & 1.0 & 7.3 & 4.0 & 20.7 \\
\hline RottM & 0.9 & 17.1 & 1.6 & 3.7 & 2.0 & 1.8 & 3.3 & 2.9 & 10.4 \\
\hline RottM & 0.7 & 11.4 & 2.3 & 3.2 & 3.6 & 2.1 & 5.8 & 3.4 & 5.1 \\
\hline RottM & 0.8 & 6.0 & 2.8 & 1.3 & 2.9 & 1.3 & 5.3 & 5.4 & 1.0 \\
\hline RottM & 0.9 & 11.3 & 4.6 & 1.7 & 3.2 & 1.4 & 5.4 & 3.7 & 16.3 \\
\hline RottM & 1.1 & 5.7 & 2.0 & 1.7 & 2.0 & 1.3 & 5.6 & 2.5 & 7.6 \\
\hline RottM & 1.2 & 9.0 & 4.1 & 2.3 & 2.1 & 5.2 & 2.0 & 2.7 & 31.7 \\
\hline RottM & 0.7 & 29.3 & 1.4 & 3.4 & 2.0 & 2.1 & 2.9 & 3.0 & 9.5 \\
\hline RottH & 2 & 72.9 & 3.4 & 4.6 & 2.6 & 2.8 & 2.2 & 2.5 & 7.0 \\
\hline RottH & 2.3 & 2.0 & 1.6 & 2.9 & 2.3 & 1.3 & 5.9 & 3.5 & 9.0 \\
\hline RottH & 3.6 & 174.9 & 4.6 & 3.2 & 1.5 & 2.3 & 3.3 & 6.5 & 11.9 \\
\hline
\end{tabular}




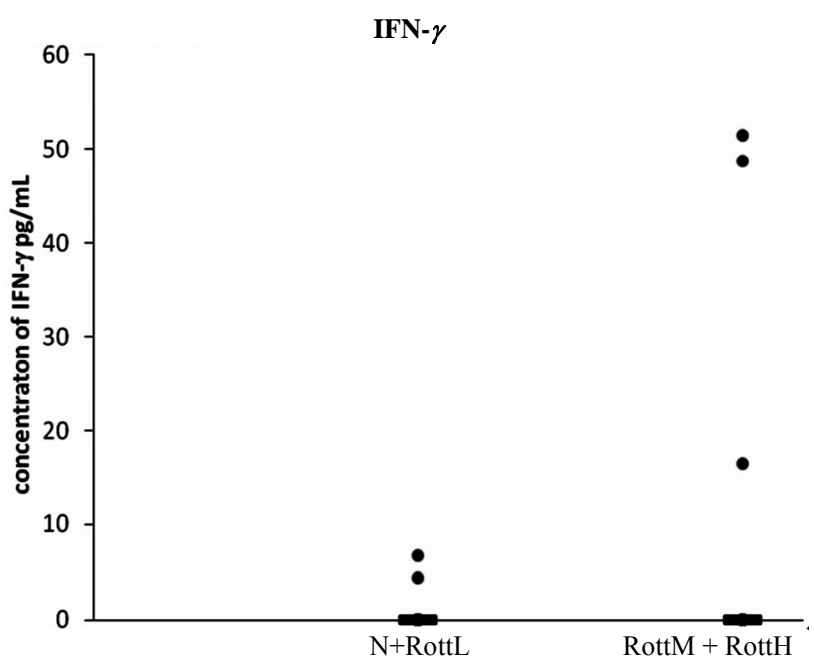

Figure 3. Plot of the concentrations of serum IFN- $\gamma$ determined by ELISA. The horizontal line corresponds to the median value of that group. There were no significant differences between normal and diseased animals in the concentration of plasma IFN $-\gamma$ quantified by ELISA.
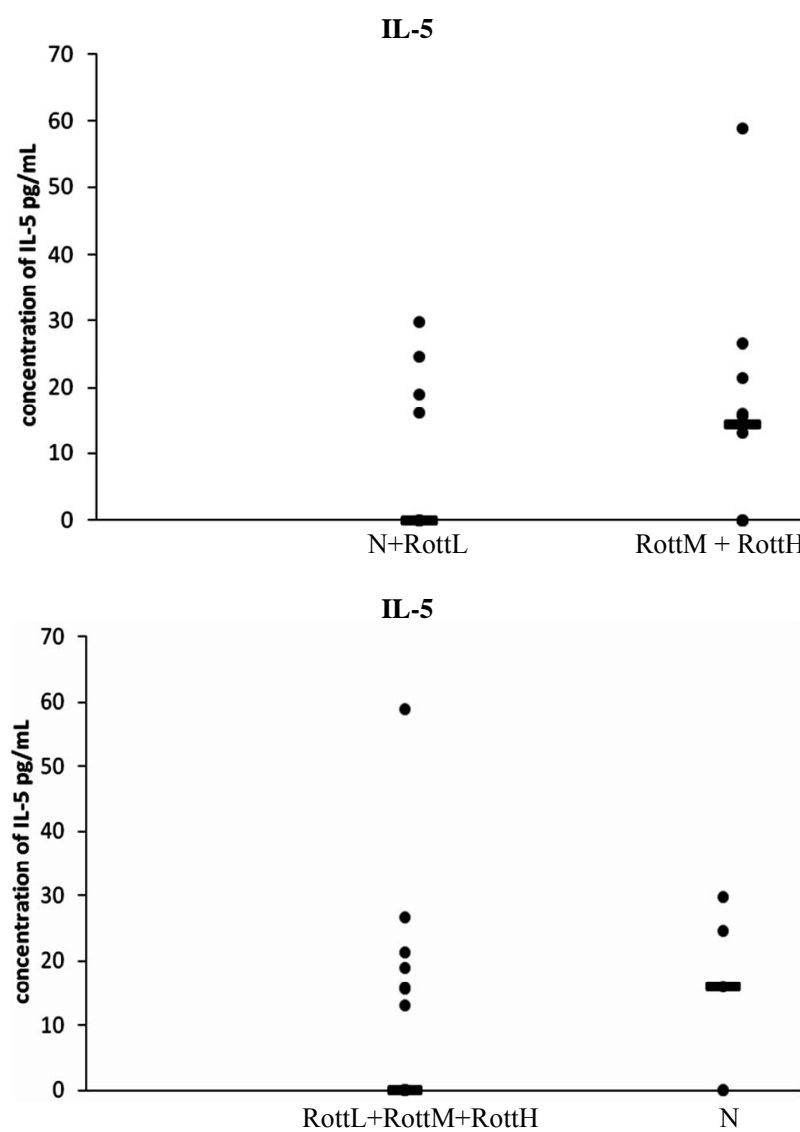

Figure 4. Plots of serum IL-5 concentrations quantified by ELISA. The horizontal line corresponds to the median value of that group. There were no significant differences between normal and diseased animals in the concentration of plasma IL-5 quantified by ELISA, or between Rottweilers and non-Rottweiler dogs. was poorly correlated with mRNA encoding IL-5 and IFN- $\gamma$ quantified through RT-PCR. The correlation coefficients for IL-5 and IFN- $\gamma$ were 0.1101 and -0.108 , respectively.

\section{Discussion}

To our knowledge, this is the first study reporting quantification of mRNA encoding cytokines in dogs with peripheral blood eosinophilia.

IFN- $\gamma$ is a pro-inflammatory cytokine that is predominantly produced by type 1 (Th1) cells and natural killer cells upon activation by IL-12 [1]. Decreased IFN- $\gamma$, and by association down-regulation of the Th1 response, may be observed with eosinophilia in people [26]? This observation suggests that IFN- $\gamma$ is unlikely to play a major role in HES, and therefore should be expressed at lower levels in dogs with eosinophilia than in those without. However, as there was no difference in IFN- $\gamma$, as determined by mRNA or ELISA, the role of IFN- $\gamma$ in canine eosinophil physiology remains unknown.

Increased IL-5 plasma concentrations have been demonstrated in a proportion of people diagnosed with HES [27]. However, this production is unrelated to aetiology (neoplastic vs. idiopathic) and is thought to be a consequence of the eosinophils themselves rather than the cause of the eosinophilia [1,2,13,27].

The lack of significant differences in mRNA and protein concentrations of IL-5 between groups in our study could be due to factors such as time of sampling and the type of samples collected. Circulating IL-5 concentrations have been observed to rise approximately two to four weeks preceding peripheral eosinophilia in some people [27], whilst episodic increases in IL-5 concentrations have been noted in others [27]. Therefore, serial sampling may have proved more useful in determining IL-5 trends in this study. It is also possible that blood concentration of IL-5 does not reflect tissue concentrations, and measurement of IL-5 in bone marrow or intestinal tissue may have produced different results. Such a level of invasive diagnostic sampling was not possible in this population of dogs. The small number of affected dogs assessed, the lack of serial sampling, and the variable clinical presentations were also factors that could have contributed to a lack of obvious differences in cytokine profiles.

It is also possible that eosinophilia in Rottweilers does not occur in response to IL-5-mediated mechanisms. Monoclonal expansion of the eosinophils themselves, independent of cytokine stimulation, has been described in people [5]. Investigation into other cytokines that stimulate eosinophil production, such as IL-3 or granulocyte-monocyte colony stimulating factor (GM-CSF) may shed more light on the immunopathogenesis of eosinophilia in dogs. 
There has been a focus on the development of antiIL-5 therapy in the management of eosinophilic disorders in man [27]. Studies assessing two different anti-IL-5 antibodies; SCH55700 (Schering-Plough, Deerfield, ILL) and mepolizumab (GlaxoSmithKline, London, UK) demonstrated effective and prolonged control of peripheral eosinophilia in people, regardless of underlying cause, disease variant, or baseline IL-5 concentration [14,27]. Although significantly increased IL-5 concentrations in our eosinophilic dogs would have lent weight to use of anti-IL-5 antibodies, normal baseline IL-5 concentrations should not preclude further investigation into its use. Additional studies are needed to determine safety, efficacy, optimal dosing, as well as observation for rebound eosinophilia in the canine model.

Eotaxins serve as chemoattractants mainly for eosinophils via inducing processes such as actin polymerization $[15,16,28]$. Eotaxins are produced by various tissue sources such as bronchial epithelial and intestinal cells and increased plasma eotaxin concentrations have been documented in people with allergic disease amongst others [29-32]. There is minimal information regarding plasma detection of eotaxins in dogs, with most veterinary studies reporting the detection and quantification of eotaxin-2 and -3 in tissue samples [17,21,33]. Inability to detect expression of eotaxin mRNA in our cohort of dogs with eosinophilia was unexpected. Furthermore, daily fluctuations in canine plasma eotaxin concentrations have not been investigated. As previously, the small number of dogs assessed, and the type of sample assessed may also have influenced the results.

\section{Conclusion}

In summary, there was no significant difference in cytokine mRNA expression (IL-4, -5, -10, -12p19, -12p35, $-12 \mathrm{p} 40,-18$, and IFN- $\gamma$ ) and quantification via ELISA of IL-5 and IFN- $\gamma$ in blood between Rottweilers with varying degrees of eosinophilia compared to Rottweiler with normal eosinophil counts and health dogs of other breeds. Additional genetic, molecular, cytokine and chemokine studies including larger numbers of affected dogs are warranted before accurate conclusions can be made about the pathogenesis of eosinophilic disease in Rottweilers.

\section{Conflicts of Interest}

None of the authors of this paper has a financial or personal relationship with other people or organisations that could inappropriately influence or bias the content of the paper.

\section{Acknowledgements}

This project was supported by the Canine Research Foundation (Australian National Kennel Club). Part of this study has been presented as a poster at the American College of Veterinary Internal Medicine Forum 2011 and as an oral abstract at the Australian and New Zealand College of Veterinary Scientists College Science Week 2011.

\section{REFERENCES}

[1] I. R. Tizard, "Veterinary Immunology: An Introduction," 7th Edition, Saunders, 2004.

[2] F. Brito-Babapulle, "Review: The Eosinophilias, including the Idiopathic Hypereosinophilic Syndrome," British Journal of Haematology, Vol. 121, No. 2, 2003, pp. 203223. doi:10.1046/j.1365-2141.2003.04195.x

[3] I. LillieHook and H. Tvedten, "Investigation of Hypereosinophilia and Potential Treatments," Veterinary Clinics of North America: Small Animal Practice, Vol. 33, No. 6, 2003, pp.1359-1378. doi:10.1016/S0195-5616(03)00097-4

[4] I. LillieHook, L. Gunnarsson, G. Zakrisson and H. Tvedten, "Diseases Associated With Pronounced Eosinophilia: A Study of 105 Dogs in Sweden," Journal of Small Animal Practice, Vol. 41, No. 6, 2000, pp. 248-253. doi:10.1111/j.1748-5827.2000.tb03934.x

[5] F. Roufosse and P. F. Weller, "Practical Approach to the Patient with Hypereosinophilia," Journal of Clinical Immunology, Vol. 126, No. 1, 2010, pp. 39-44. doi:10.1016/j.jaci.2010.04.011

[6] C. Clercx, D. Peeters, F. Snaps, P. Hansen, K. McEntee, J. Detilleux, M. Henroteaux and M. J. Day, "Eosinophilic Bronchopneumopathy in Dogs," Journal of Veterinary Internal Medicine, Vol. 14, No. 3, 2000, pp. 282-291. doi:10.1111/j.1939-1676.2000.tb01168.x

[7] I. Aroch, S. Perl and A. Markovics, "Disseminated Eosinophilic Disease Resembling Idiopathic Hypereosinophilic Syndrome in a Dog," The Veterinary Record, Vol. 149 , No. 13,2001 , pp. 386-389.

doi:10.1136/vr.149.13.386

[8] M. C. Perkins and A. D. J. Watson, "Successful Treatment of Hypereosinophilic Syndrome in a Dog," Australian Veterinary Journal, Vol. 79, No. 10, 2001, pp. 686689. doi:10.1111/j.1751-0813.2001.tb10671.x

[9] A. J. German, D. J. Holden, E. J. Hall and M. J. Day, "Eosinophilic Diseases in Two Cavelier King Charles Spaniels," Journal of Small Animal Practice, Vol. 43, No. 12,2002 , pp. 533-538. doi:10.1111/j.1748-5827.2002.tb00026.x

[10] G. D. Bellou, S. Kleinschmidt, F. Meneses, I. Nolte and M. Hewicker-Trautwein, "Eosinophilic Granulomatous Gastroenterocolitis and Hepatitis in a 1-Year-Old Male Siberian Husky," Veterinary Pathology, Vol. 43, No. 6, 2006, pp. 1022-1025. doi:10.1354/vp.43-6-1022

[11] F. E. James and C. S. Mansfield, "Clinical Remission of Idiopathic Hypereosinophilic Syndrome in a Rottweiler," Australian Veterinary Journal, Vol. 87, No. 8, 2009, pp. 330-333. doi:10.1111/j.1751-0813.2009.00461.x

[12] J. E. Sykes, D. J. Weiss, L. C. Buoen, M. M. Blauvelt, 
and D. W. Hayden, "Idiopathic Hypereosinophilic Syndrome in 3 Rottweilers," Journal of Veterinary Internal Medicine, Vol. 15, No. 2, 2001, pp. 162-166. doi:10.1111/j.1939-1676.2001.tb01251.x

[13] A. D. Klion, B. S. Bochner, G. J. Gleich, T. B. Nutman, M. E. Rothenberg, H. U. Simon, M. E. Wechsler and P. F. Weller: The Hypereosinophilic Syndromes Working Group, "Approaches to the Treatment of Hypereosinophilic Syndromes: A Workshop Summary Report," Journal of Allergy and Clinical Immunology, Vol. 117, No. 6, 2009, pp. 1292-1301. doi:10.1016/i.jaci.2006.02.042

[14] W. W. Busse, J. Ring, J. Huss-Marp and J. E. Kahn, “A Review of Treatment with Mepolizumab, an Anti-IL-5 mAb, in Hypereosinophilic Syndromes and Asthma," Journal of Allergy and Clinical Immunology, Vol. 125, No. 4, 2010, pp. 803-813. doi:10.1016/j.jaci.2009.11.048

[15] J. Elsner, H. Petering, C. Kluthe, D. Kimmig, R. Smolarski, P. Ponath and A. Kap, "Eotaxin-2 Activates Chemotaxis-Related Events and Release of Reactive Oxygen Species via Pertussis Toxin-Sensitive G Proteins in Human Eosinophils," European Journal of Immunology, Vol. 28, No. 7, 1998, pp. 2152-2158. doi:10.1002/(SICI)1521-4141(199807)28:07<2152::AIDIMMU2152>3.0.CO;2-G

[16] A. Shinkai, H. Yoshisue, M. Koike, E. Shoji, S. Nakagawa, A. Saito, T. Takeda, S. Imabeppu, Y. Kato, N. Hanai, H. Amazawa, T. Kuga and T. Nishi, "A Novel Human CC Chemokine, Eotaxin-3, Which Is Expressed in IL-4-Stimulated Vascular Endothelial Cells, Exhibits Potent Activity Toward Eosinophils," Journal of Immunology, Vol. 163, No. 3, 1999, pp. 1602-1610.

[17] N. Zimmermann, S. P. Hogan, A. Mishra, E. B. Brandt, T. R. Bodette, S. M. Pope, F. D. Finkelman and M. E. Rothenberg, "Murine Eotaxin-2: A Constitutive Eosinophil Chemokine Induced by Allergen Challenge and IL-4 Overexpression," Journal of Immunology, Vol. 165, No. 10, 2000, pp. 5839-5846.

[18] S. Rozen and H. Skaletsky, "Primer 3 on the www for General Users and for Biologist Programmers," Methods in Molecular Biology, Vol. 132, 2000, pp. 365-386.

[19] I. R. Peters, C. R. Helps, E. J. Hall and M. J. Day, "Real-time RT-PCR: Considerations for Efficient and Sensitive Assay Design," Journal of Immunological Methods, Vol. 286, No. 1-2, 2004, pp. 203-217. doi:10.1016/j.jim.2004.01.003

[20] I. R. Peters, C. R. Helps, E. L. Calvert, E. J. Hall and M. J. Day, "Cytokine mRNA Quantification in Histologically Normal Canine Duodenal Mucosa by Real-time RTPCR," Veterinary Immunology and Immunopathology, Vol. 103, No. 1-2, 2005, pp. 101-111. doi:10.1016/j.vetimm.2004.08.020

[21] D. Peeters, I. R. Peters, F. Farnir, C. Clercx and M. J. Day, "Real-Time RT-PCR Quantification of mRNA Encoding Cytokines and Chemokines in Histologically Normal Canine Nasal, Bronchial and Pulmonary Tissue," Veterinary Immunology and Immunopathology, Vol. 104, No. 3-4, 2005, pp. 195-204. doi:10.1016/j.vetimm.2004.11.007

[22] D. Peeters, I. R. Peters, C. Clerex and M. J. Day, "RealTime RT-PCR Quantification of mRNA Encoding Cyto- kines, CC Chemokines and CCR3 in Bronchial Biopsies from Dogs with Eosinophilic Bronchopneumopathy," $V e$ terinary Immunology and Immunopathology, Vol. 110, No. 1-2, 2006, pp. 65-77.

doi:10.1016/j.vetimm.2005.09.004

[23] D. Peeters, I. R. Peters, C. R. Helps, A. Gabriel, M. J. Day and C. Clercx, "Distinct Tissue Cytokine and Chemokine mRNA Expression in Canine Sino-Nasal Aspergillosis and Idiopathic Lymphoplasmacytic Rhinitis," Veterinary Immunology and Immunopathology, Vol. 117, No. 1-2, 2007, pp. 95-105. doi:10.1016/j.vetimm.2007.01.018

[24] I. R. Peters, D. Peeters, C. R. Helps and M. J. Day, "Development and Application of Multiple Internal Reference (Housekeeper) Gene Assays for Accurate Normalisation of Canine Gene Expression Studies," Veterinary Immunology and Immunopathology, Vol. 117, No. 1-2, 2007, pp. 55-66. doi:10.1016/j.vetimm.2007.01.011

[25] J. Vandesompele, K. De Preter, F. Pattyn, B. Poppe, N. Van Roy, A. De Paepe and F. Speleman, "Accurate Normalization of Real-Time Quantitative RT-PCR Data by Geometric Averaging of Multiple Internal Control Genes," Genome Biology, Vol. 3, No. 7, 2002, Article ID: RESEARCH0034.

[26] A. Meager, "The Interferons: Characterization and Application," Wiley-VCH, Verlag Gmbh and Co. KGaA, Weinheim, 2006.

[27] A. D. Klion, M. A. Law, P. Noel, Y. J. Kim, T. P. Haverty and T. B. Nutman, "Safety and Efficacy of the Monoclonal Anti-Interleukin-5 Antibody SCH55700 in the Treatment of Patients with Hypereosinophilic Syndrome," Blood, Vol. 103, No. 8, 2004, pp. 2939-2941. doi:10.1182/blood-2003-10-3620

[28] A. N. Matthews, D. S. Friend, N. Zimmermann, M. N. Sarafi, A. D. Luster, E. Perlman, S. E. Wert and M. E. Rothenberg, "Eotaxin is Required for the Baseline Level of Tissue Eosinophils," Proceedings of the National Academy of Sciences of the United States of America, Vol. 95, No. 11, 1998, pp. 6273-6278. doi:10.1073/pnas.95.11.6273

[29] A. Mir, M. Minguez, J. Tatay, I. Pascual, A. Pena, V. Sanchiz, P. Almela, F. Mora and A. Benages, "Elevated Serum Eotaxin Levels in Patients with Inflammatory Bowel Disease," American Journal of Gastroenterology, Vol. 97, No. 6, 2002, pp.1452-1457. doi:10.1111/j.1572-0241.2002.05687.x

[30] A. Komiya, H. Nagase, H. Yamada, T. Sekiya, M. Yamaguchi, Y. Sano, N. Hanai, A. Furuya, K. Ohta, K. Matsushima, O. Yoshie, K. Yamamoto and K. Harai, "Concerted Expression of Eotaxin-1, Eotaxin-2, and Eotaxin-3 in Human Bronchial Epithelial Cells," Cellular Immunology, Vol. 225, No. 2, 2003, pp. 91-100. doi:10.1016/j.cellimm.2003.10.001

[31] T. Targowski, K. Jahnz-Rózyk, T. Plusa and E. Glodzinska-Wyszogrodzka, "Influence of Age and Gender on Serum Eotaxin Concentration in Healthy and Allergic People," Journal of Investigational Allergology and Clinical Immunology, Vol. 15, No. 4, 2005, pp. 277-282.

[32] K. Polzer, T. Karonitsch, T. Neumann, G. Eger, C. Haberler, A. Soleiman, B. Hellmich, E. Csernok, J. 
Distler, B. Manger, K. Redlich, G. Schett and J. Zwerina, "Eotaxin-3 is Involved in Churg-Strauss Syndrome-A Serum Marker Closely Correlating with Disease Activity," Rheumatology, Vol. 47, No. 6, 2008, pp. 804-808. doi:10.1093/rheumatology/ken033

[33] B. Vogel, A. Klinder, D. Sittig and G. Aust, "Bovine
Eotaxin (CCL11) - An Unusual Member of the Eotaxin Group-Attracts Eosinophils in Vitro but is Not Responsible for Eosinophilia in the Ovary," Veterinary Immunology and Immunopathology, Vol. 107, No. 1-2, 2005, pp. 67-77. doi:10.1016/j.vetimm.2005.03.013

Supplementary Table A1. Primer and probe sequences used for quantitative RT-PCR.

\begin{tabular}{|c|c|c|c|c|}
\hline Primer & Product & Forward Primer & Reverse Primer & Probe \\
\hline Set & igth & $\left(5^{\prime}-3^{\prime}\right)$ & $\left(5^{\prime}-3^{\prime}\right)$ & $\left(5^{\prime}-3 ’\right)$ \\
\hline G3PDH & 90 & $\begin{array}{l}\text { TCAACG- } \\
\text { GATTTGGCCGTATTGG }\end{array}$ & TGAAGGGGTCATTGATGGCG & $\begin{array}{c}\text { TexasRed-CAGGGCTGCTTTTAACTCTG } \\
\text { GCAAAGTGGA-BHQ-2 }\end{array}$ \\
\hline HMBS & 112 & TCACCATCGGAGCCATCT & GTTCCCACCACGCTCTTCT & $\begin{array}{l}\text { FAM-CAGCATCATAGGGGTTCTCCCGC-B } \\
\text { HQ-1 }\end{array}$ \\
\hline HPRT1 & 123 & CACTGGGAAAACAATGCAGA & $\begin{array}{l}\text { ACAAAGTCAGGTTTA- } \\
\text { TAGCCAACA }\end{array}$ & $\begin{array}{l}\text { FAM-TGCTGGTGAAAAGGACCCCTCG-BH } \\
\text { Q-1 }\end{array}$ \\
\hline RPL13A & 87 & GCCGGAAGGTTGTAGTCGT & GGAGGAAGGCCAGGTAATTC & $\begin{array}{c}\text { FAM-TGTGAAGGCATCAACATTTCTGGC } \\
\text { A-BHQ-1 }\end{array}$ \\
\hline RPS18 & 116 & TGCTCATGTGGTATTGAGGAA & TCTTATACTGGCGTGGATTCTG & $\begin{array}{l}\text { FAM-CACTGAGGATGAGGTGGAACGTGT } \\
\text { G-BHQ-1 }\end{array}$ \\
\hline SDHA & 92 & GCCTTGGATCTCTTGATGGA & TTCTTGGCTCTTATGCGATG & $\begin{array}{l}\text { FAM-TGTCGTGGTGTTATTGCACTGTGCA- } \\
\text { BHQ-1 }\end{array}$ \\
\hline TBP & 96 & $\begin{array}{l}\text { CTATTTCTTGGTGTGCAT- } \\
\text { GAGG }\end{array}$ & CCTCGGCATTCAGTCTTTTC & $\begin{array}{l}\text { FAM-TCGCTAAGAAGCGTGTACTGGGGA } \\
\text { TG-BHQ-1 }\end{array}$ \\
\hline YWHAZ & 94 & CGAAGTTGCTGCTGGTGA & TTGCATTTCCTTTTTGCTGA & $\begin{array}{c}\text { FAM-AGGGATTGTGGATCAGTCACAACA } \\
\text { AGC-BHQ-1 }\end{array}$ \\
\hline IL-4 & 123 & GCTCCAAAGAACACAAGCGA & CATGCTGCTGAGGTTCCTGT & $\begin{array}{c}\text { Texas Red-TGCAGAGCTGCTACTGTACTG } \\
\text { CGGC-ELLE }\end{array}$ \\
\hline IL-5 & 158 & GACTGGTGGCAGAGACCTTGA & CGTGGGCAGTTTGGTTCTTC & $\begin{array}{l}\text { FAM-CGAACTTGGCTGATAGGCGATGG-B } \\
\text { HQ-1 }\end{array}$ \\
\hline IL-10 & 101 & CGACCCAGACATCAAGAACC & CACAGGGAAGAAATCGGTGA & $\begin{array}{l}\text { FAM-TCCCTGGGAGAGAAGCTCAAGACC } \\
\text { C-BHQ-1 }\end{array}$ \\
\hline IL-12p19 & 108 & GTGATCCCCAAGGACTCAAA & GCTCCCCTGTGAAAATGTCT & $\begin{array}{l}\text { FAM-AAAGGATCCACCAGGGCCTGG-BH } \\
\text { Q-1 }\end{array}$ \\
\hline IL-12p35 & 146 & CGTCAGCAACACGCTTCA & $\begin{array}{l}\text { AACTCTCATTCATGGTTAATTC- } \\
\text { CAG }\end{array}$ & $\begin{array}{c}\text { FAM-CCAGCACAGTGGAGGCCTGC-BHQ- } \\
1\end{array}$ \\
\hline IL-12p40 & 109 & $\begin{array}{l}\text { CAGCAGAGAGGGTCA- } \\
\text { GAGTGG }\end{array}$ & ACGACCTCGATGGGTAGGC & $\begin{array}{l}\text { FAM-TGGAGTGTCAGGAGGGCAGTGC-B } \\
\text { HQ-1 }\end{array}$ \\
\hline IL-18 & 139 & $\begin{array}{l}\text { TTAAAGCGGAAAGTGAT- } \\
\text { GAAGG }\end{array}$ & TCGGGCATATCCTCAAATACA & $\begin{array}{l}\text { Texas Red-GAAATTTGAACGAC } \\
\text { CAAGTCCTCTTCG-ELLE }\end{array}$ \\
\hline IFN- $\gamma$ & 113 & TCAACCCCTTCTCGCCACT & GCTGCCTACTTGGTCCCTGA & FAM-CCCCACCCGAACCTCTTCCT-BHQ-1 \\
\hline Eotaxin-2 & 95 & AAGGCAGGAGTGGTCTTCAC & $\begin{array}{l}\text { GCCTCTATGTTCTTCAT- } \\
\text { CAACTTCT }\end{array}$ & $\begin{array}{c}\text { FAM-TGTGGCGATCCCCAGCTGCA-BHQ- } \\
1\end{array}$ \\
\hline Eotaxin-3 & 128 & TCTGCTGCTTCCAATATAGCC & TGCACAGACTTTATGGCCTTT & $\begin{array}{c}\text { FAM-AGCTGCTCCCAGCAGGCTGTGATA } \\
\text { T-BHQ-1 }\end{array}$ \\
\hline
\end{tabular}

